\title{
Commentary
}

\section{The fifth generation}

Expert systems, artificial intelligence and knowledge engineering are terms that are often used interchangeably. They each describe the one approach that we now have for solving nonmathematical problems. This approach depends upon a knowledge engineer being able to extract information from a human expert and translating it into rules that can be put into software. There are numerous existing systems in use, particularly in the fields of medical diagnosis, geology, chemical structures, civil engineering, etc. There is advanced research in management applications of expert systems.

Possibly the best evidence of the potential of this technique can be seen in Japan's Fifth Generation Computer Project.

In 1979, the Japanese commercial and academic bodies set up a series of committees, under the guidance of the Ministry of International Trade and Industry, with the aim of studying the possibile contribution of advanced technology to the solution of a country's economic and social problems in 1990s, particularly the role to be played by the fifth generation of computers. They defined the strategic problems that have to be solved, viz. increase productivity in low productivity areas, save energy and meet international competition while striving for co-operation.

To achieve these goals, it is necessary to develop a very advanced type of computer that will operate on spoken language, to eliminate the current programming languages, and to be able to perform inferential logic operations in addition to conventional data processing.

We are moving towards a new software environment, one with many dimensions. The idea was launched in Japan, but today it is analyzed everywhere.

From the first generation of computers pro- grammed in machine code, through the second generation based on assembly languages, and the third generation defined by procedure-oriented high order language compilers, to the fourth generation defined by query driven high level language program generators, the way was paved for the fifth generation, expert knowledge-based systems.

Knowledge systems differ from traditional computer programs in a variety of ways. Based on radically different system architectures and programming styles, the most visible departure from standard computer systems is the capability to interact intelligently with their users.

The man-machine relation is dominant. The technical system is impregnated by the human system.

Discussions of the fifth generation project usually focus on the changes that have been taking place within computer science itself. But we can also look at changes in that discipline that deals with the users. Like changes in computer sciences, changes in the last two decades in human systems management have indeed been dramatic.

In a preface to a recent book devoted to the foundations of decision support systems, Herbert Simon notes that the major force driving the gradual enlargement of aids to decision making is the nature of decision making itself. Nothing is more true.

What is happening now in computer science has an explanation partly given by human systems management. $H S M$ is open to such debates. The challenge of the Fifth Generation has been and can be analyzed. The huge community of decision makers needs a forum where the implication of a new and exciting technology can be discussed and which is devoted to narrowing the gap between users and computers.

This Journal can be such a forum. Let us use it.

Constantin V. NEGOIŢǍ Hunter College, CUNY

North-Holland

Human Systems Management 5 (1985) 259 